ARTICLE

\title{
Dimer rattling mode induced low thermal conductivity in an excellent acoustic conductor
}

Ji Qi ${ }^{1,2}$, Baojuan Dong (1) 1, Zhe Zhang ${ }^{1,2}$, Zhao Zhang ${ }^{1,2}$, Yanna Chen ${ }^{3}$, Qiang Zhang (1) ${ }^{4}$, Sergey Danilkin ${ }^{5}$, Xi Chen ${ }^{6,7}$, Jiaming He (1) ${ }^{6}$, Liangwei Fu ${ }^{8}$, Xiaoming Jiang ${ }^{9}$, Guozhi Chai (1) ${ }^{10}$, Satoshi Hiroi ${ }^{3}$, Koji Ohara (1) ${ }^{11}$, Zongteng Zhang ${ }^{1,2}$, Weijun Ren', Teng Yang (1) 1,2, Jianshi Zhou ${ }^{6}$, Sakata Osami (iD ${ }^{3}$, Jiaqing $\mathrm{He}^{8}$, Dehong Yu (i) ${ }^{5 凶}$, Bing Li (i) ${ }^{1,2 \otimes} \&$ Zhidong Zhang (i) ${ }^{1,2}$

A solid with larger sound speeds usually exhibits higher lattice thermal conductivity. Here, we report an exception that $\mathrm{CuP}_{2}$ has a quite large mean sound speed of $4155 \mathrm{~m} \mathrm{~s}^{-1}$, comparable to GaAs, but single crystals show very low lattice thermal conductivity of about $4 \mathrm{~W} \mathrm{~m}^{-1} \mathrm{~K}^{-1}$ at room temperature, one order of magnitude smaller than GaAs. To understand such a puzzling thermal transport behavior, we have thoroughly investigated the atomic structures and lattice dynamics by combining neutron scattering techniques with first-principles simulations. This compound crystallizes in a layered structure where $\mathrm{Cu}$ atoms forming dimers are sandwiched in between $\mathrm{P}$ atomic networks. In this work, we reveal that $\mathrm{Cu}$ atomic dimers vibrate as a rattling mode with frequency around $11 \mathrm{meV}$, which is manifested to be remarkably anharmonic and strongly scatters acoustic phonons to achieve the low lattice thermal conductivity.

\footnotetext{
${ }^{1}$ Shenyang National Laboratory for Materials Science, Institute of Metal Research, Chinese Academy of Sciences, 72 Wenhua Road, Shenyang 110016, China. ${ }^{2}$ School of Materials Science and Engineering, University of Science and Technology of China, Shenyang 110016, China. ${ }^{3}$ Synchrontron X-ray station at SPring-8, Research Network and Facility Services Division, National Institute for Materials Science (NIMS), 1-1-1 Kouto, Sayo-Cho, Sayo-Gun, Hyogo 6795148, Japan. ${ }^{4}$ Spallation Neutron Source, Oak Ridge National Laboratory, Oak Ridge, TN 37831, USA. ${ }^{5}$ Australian Nuclear Science and Technology Organisation, Locked Bag 2001, Kirrawee, DC NSW 2232, Australia. ${ }^{6}$ Department of Mechanical Engineering, University of Texas at Austin, Austin, TX 78712, USA. ${ }^{7}$ Department of Electrical and Computer Engineering, University of California, Riverside, CA 92521, USA. ${ }^{8}$ Department of Physics, Southern University of Science and Technology, Shenzhen 518005, China. ${ }^{9}$ State Key Laboratory of Structural Chemistry, Fujian Institute of Research on the Structure of Matter, Chinese Academy of Sciences, Fuzhou, Fujian 350002, China. ${ }^{10}$ Key Lab for Magnetism and Magnetic Materials of the Ministry of Education, Lanzhou University, Lanzhou 730000, China. ${ }^{11}$ SPring-8, Diffraction and Scattering Division, Japan Synchrotron Radiation Research Institute, 1-1-1 Kouto,

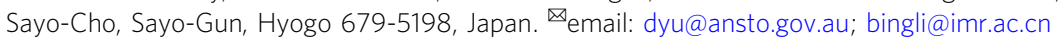


$\mathrm{T}$ hermal conduction is one of the most fundamental physical properties of materials ${ }^{1}$. Materials with low thermal conductivity are desirable for a great variety of applications such as thermal insulation ${ }^{2}$, phase transition memory devices ${ }^{3}$, and thermoelectric energy conversion ${ }^{4}$. In electrically insulating nonmagnetic systems, phonons are the major heat carrier, and lattice thermal conductivity is proportional to the product of the square of sound speeds and phonon lifetimes ${ }^{5-7}$. To decrease the thermal conductivity, a general approach is to reduce the phonon lifetimes through enhancing phonon-phonon and phonondisorder scattering. Phonon-phonon scattering, also known as phonon anharmonic interactions, dominates the thermal conductivity of disorder-free systems like $\mathrm{PbTe}^{8}$ and $\mathrm{SnSe}^{9}$. There is a special case called rattling, i.e., a localized vibrational mode scattering acoustic phonons with an indication of anti-crossing points in the phonon dispersions ${ }^{10}$. This idea has been broadly applied to rationalize the low thermal conductivity in phononglasses thermoelectric materials like filled skutterudites and clathrates ${ }^{11-13}$. In addition, spatially hierarchical atomic disorder has also been widely used, including solid solutions of alloys ${ }^{14}$, nanostructures ${ }^{15,16}$, liquid-like disorder ${ }^{17,18}$ and so forth, to lower the thermal conductivity through phonon-disorder scattering. For a variety of disorder-free compounds, we account for the relationship between thermal conductivity and sound speeds in Fig. 1a $\left(\right.$ ref. $\left.{ }^{19}\right)$. In the logarithmic scale, most of the compounds are distributed around an empirical straight line. For a given thermal conductivity value the sound speeds are the dominating factor for materials above the line while the lattice anharmonicity governs thermal transport of materials below the line. Located in the line, single-crystalline diamond ${ }^{20,21}$ has the highest lattice thermal conductivity among those materials, $2400 \mathrm{~W} \mathrm{~m}^{-1} \mathrm{~K}^{-1}$, accommodating a very high mean sound speed of $14,400 \mathrm{~m} \mathrm{~s}^{-1}$. GaAs is also situated near the line with a mean sound speed of $3627 \mathrm{~m} \mathrm{~s}^{-1}$, whereas $\mathrm{CuP}_{2}$ has a similar mean sound speed, but its thermal conductivity is almost ten times lower 22,23 .

The temperature dependencies of lattice thermal conductivity $\left(\kappa_{\text {lat }}\right)$ of $\mathrm{CuP}_{2}$ single crystals along $b c$ plane and out of $b c$ plane are shown in Fig. $1 \mathrm{~b}$ in the temperature region from 50 to $300 \mathrm{~K}$, respectively. The $\kappa_{\text {lat }}$ values in both directions increase as temperature decreases. At $300 \mathrm{~K}, \kappa_{\text {lat }}$ along the $b c$ plane is $4.66 \mathrm{~W} \mathrm{~m}^{-1}$ $\mathrm{K}^{-1}$, while $3.57 \mathrm{~W} \mathrm{~m}^{-1} \mathrm{~K}^{-1}$ out of the $b c$ plane. $\kappa_{\text {lat }}$ for a polycrystalline sample is shown in Supplementary Fig. 1. At room temperature, it is about $0.62 \mathrm{~W} \mathrm{~m}^{-1} \mathrm{~K}^{-1}$. This value is greatly reduced compared to the single crystals, which might be attributed to the grain boundaries and defects scattering ${ }^{24}$. In order to understand the mechanism responsible for such a low thermal conductivity in $\mathrm{CuP}_{2}$, we conducted a systematical study of the atomic structure and lattice dynamics of this material.

\section{Results}

Atomic structures. This compound crystallizes in a relatively simple monoclinic structure with space group $P 2_{1} / c$ (ref. ${ }^{25}$ ). As shown in Fig. 1c, the structure is characteristic of a layered configuration where $\mathrm{Cu}$ layers (spacing $1.59 \AA$ ) and $\mathrm{P}$ network (spacing $3.85 \AA$ ) repeat alternatively along the $a$-axis. The $\mathrm{Cu}$ atoms forming a dimer occupy an equivalent position. The arrows in orange represent the vibration directions of $\mathrm{Cu}$ atoms associated with the rattling mode to be described later. As shown in Fig. $1 c, d$, the intra-dimer distance is $2.48 \AA$, while the interdimer distances are $3.85,4.81$, and $7.53 \AA$ along $a, b$, and $c$ directions, respectively. The electron localization function (ELF) calculation was used to support the weak bonding nature between
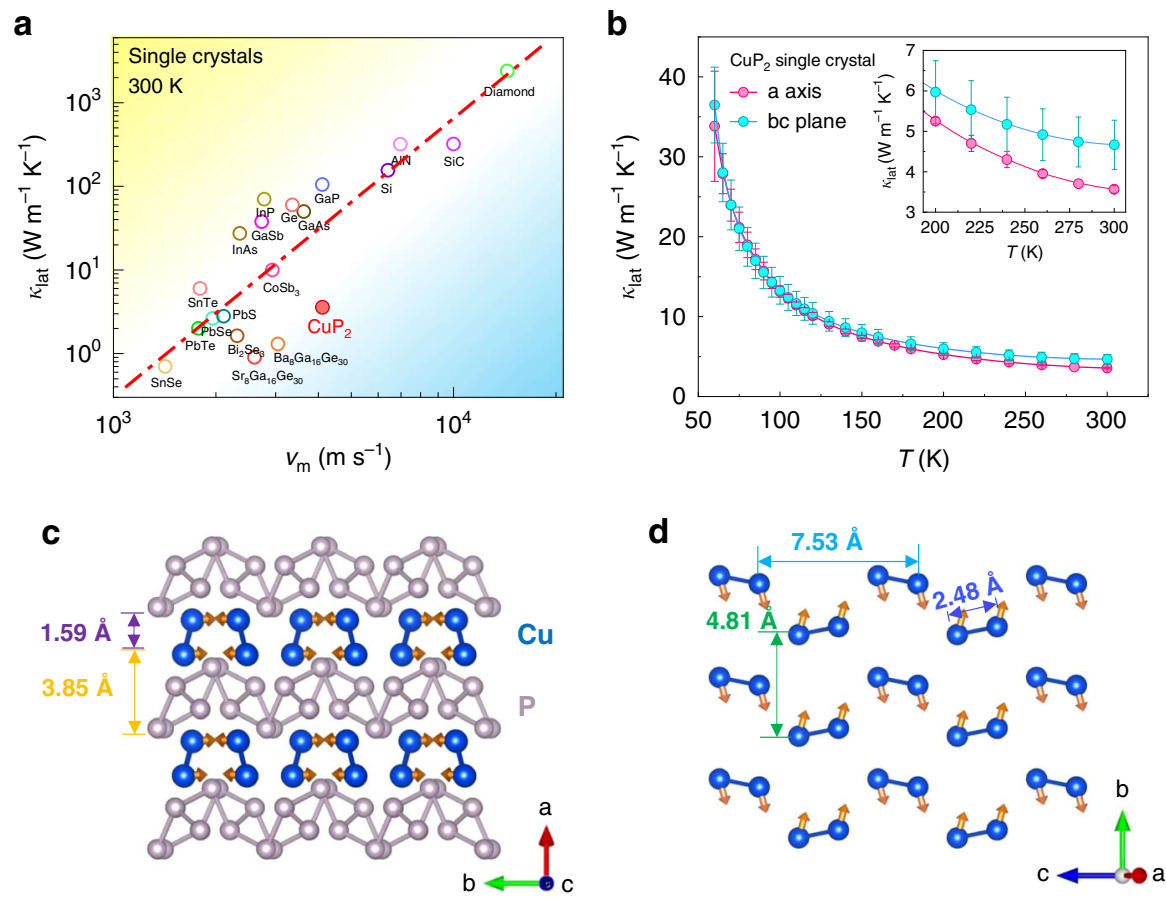

Fig. 1 Lattice thermal conductivity and crystal structure of $\mathbf{C u P}_{\mathbf{2}}$. a A survey of lattice thermal conductivity of disorder-free materials vs. mean sound speed $v_{m}$. The detailed data are listed in Supplementary Table 1 (for polycrystalline samples, please refer to Supplementary Fig. 1 and Table 2). b Temperature dependence of the lattice thermal conductivity of the $\mathrm{CuP}_{2}$ single crystal is shown from 50 to $300 \mathrm{~K}$ measured by a steady-state comparative method. The inset shows the data near room temperature. The error bars indicate the uncertainty (see "Methods"). c The layered structure with $\mathrm{Cu}$ dimer layers and $\mathrm{P}$ network layers are highlighted. The arrows in orange on $\mathrm{Cu}$ atoms represent the vibrational directions at the $\Gamma$ point of the optical phonon mode located at about $11 \mathrm{meV}$ (see Fig. 4). The atomic motions of this mode are also displayed in Supplementary Movie 1. d The isolated Cu dimer layers with intra- and inter-dimer distances labeled. 
a
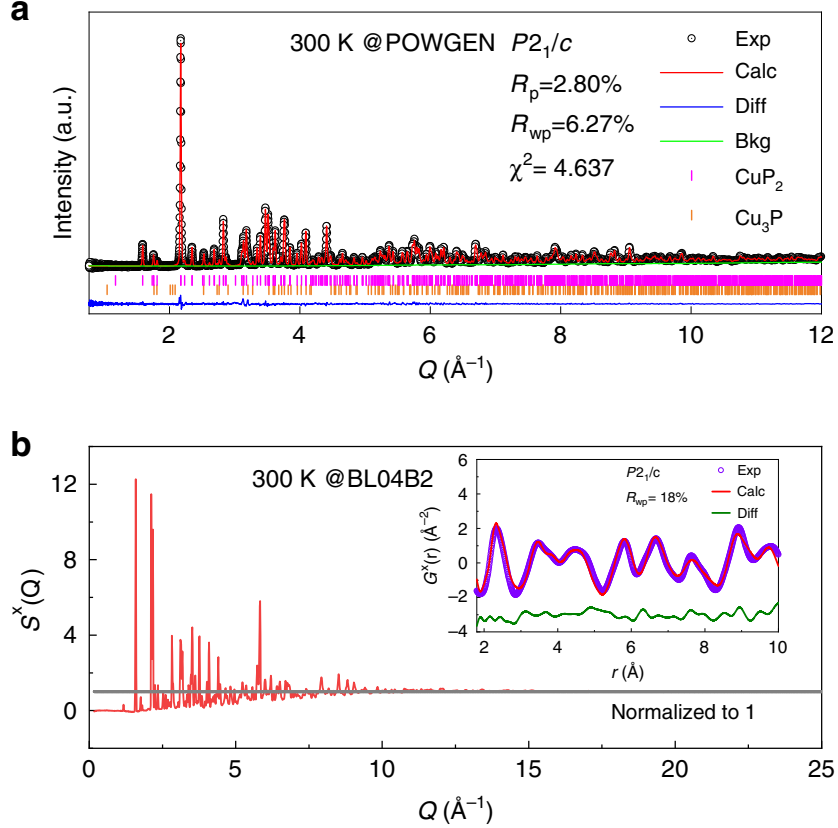

Fig. 2 Average and local structures of $\mathbf{C u P}_{\mathbf{2}}$. a Neutron powder diffraction pattern and Rietveld refinement analysis at $300 \mathrm{~K}$. b The structure factor, $S^{X}(Q)$, obtained in $X$-ray total scattering at $300 \mathrm{~K}$. The inset is the reduced PDF, $G^{X}(r)$, as well as the real-space refinement based on the $P 2_{1} / c$ crystal model.

$\mathrm{Cu}$ dimers as a direct theoretical evidence (see Supplementary Fig. 2). ELF around $\mathrm{Cu}$ atoms is around 0.2 , which suggests a weak metallic bond between $\mathrm{Cu}$ atoms within $\mathrm{Cu}$ dimers, while ELF is almost zero between $\mathrm{Cu}$ dimers. This distinction along with the calculated charge density well supports the dimer nature. In consideration of the isolated nature of the $\mathrm{Cu}$ dimer, the vibration mode of the dimer as a whole unit can be treated as a localized phonon. Later we will show that these localized phonons play a crucial role in suppressing the thermal conduction of $\mathrm{CuP}_{2}$.

Our neutron powder diffraction results confirm the crystal structure as previously reported ${ }^{25}$. Shown in Fig. $2 \mathrm{a}$ is the neutron powder diffraction pattern at room temperature (for data at lower temperatures, refer to Supplementary Fig. 3). The Rietveld refinement analysis suggests that the majority phase is monoclinic $\mathrm{CuP}_{2}$ while there is a minority phase of $\mathrm{Cu}_{3} \mathrm{P}$, whose volumetric fraction is only $2.79 \%$. This powder sample, crushed from single crystals, is strongly textured along (100) because of the excellent ductility associated with the layered lattice structure. It is important to notice that the background of the diffraction data is quite flat with no obvious diffuse scattering observed, which indicates no discernable atomic disorder in the sample. The detailed structural parameters determined in the refinements are listed in Supplementary Table 3. The Debye-Waller factors, in particular, $U_{22}$ and $U_{33}$ of $\mathrm{Cu}$ atoms are much larger than those of $\mathrm{P}$ atoms. This indicates that there is a significant thermal motion of $\mathrm{Cu}$ atoms confined within the layers. The full temperature dependencies of the lattice parameters and Debye-Waller factors are summarized in Supplementary Fig. 4. It should be noted that the fitting of temperature dependencies of Debye-Waller factors to the Einstein model suggests there is negligible atomic disorder. In addition to the powder diffraction, our single-crystal X-ray diffraction also confirms the structure. While the average structural study suggests the right phase and negligible disorder, we further employ the pair distribution function (PDF) analysis to directly confirm the disorder-free nature of the system. As shown in Fig. 2b, the X-ray structure factor $S^{\mathrm{X}}(Q)$ is well normalized to 1 at high $Q$ at $300 \mathrm{~K}$. Reduced PDF, $G^{\mathrm{X}}(r)$, is subsequently obtained via Fourier transform of $S^{\mathrm{X}}(Q)$. The inset of Fig. 2b presents the experimental $G^{\mathrm{X}}(r)$ that is well reproduced by the $P 2_{1} / c$ crystal model, supporting the disorder-free nature.

Lattice dynamics. The above structural study excludes disorder scattering as an origin of the low thermal conductivity. Now, we move to the lattice dynamics study for lattice anharmonicity. We begin with a survey over a large reciprocal space of the dynamic structure function $S(\mathbf{Q}, E)$ vs. momentum transfer $(\mathbf{Q})$ and energy transfer $(E)$ using the time-of-flight neutron spectrometer-Pelican. The measurements are carried out in the scattering plane defined by $[H, 0,0]$ and $[0, L, L]$. Shown in Fig. $3 \mathrm{a}$ is the phonon dispersion along $[H, H, H]$ direction of the Brillouin zone $\mathbf{G}=(111)$. The branches of acoustic phonons are clearly observed, in agreement with the results from the triple-axis spectrometer-Taipan and the density functional theory (DFT) calculations. Unfortunately, the optical phonon modes above $10 \mathrm{meV}$ cannot be observed due to limited accessible energy range and energy resolution. For more details of dispersions of different Brillouin zones, please refer to Supplementary Fig. 5. Before we rationally perform detailed INS measurements at Taipan, the lattice dynamics are fully exploited using DFT calculations. The DFT calculated phonon dispersions of Brillouin zones $\mathbf{G}=(200), \mathbf{G}=(022)$, and $\mathbf{G}=$ (111) are plotted in Fig. $3 \mathrm{~b}$ up to $16 \mathrm{meV}$ while the complete dispersions are plotted in Supplementary Fig. 6. We can see the steep acoustic phonon branches that originate from the individual $\Gamma$ point and reach the zone boundaries at about $10 \mathrm{meV}$. Above the acoustic phonon branches, there are a few flat optical phonon bands up to $16 \mathrm{meV}$.

Then, we focus on detailed investigations by conducting constant- $E$ as well as constant- $\mathbf{Q}$ scans for Brillouin zones of $\mathbf{G}=$ (200), $\mathbf{G}=$ (022), and $\mathbf{G}=$ (111) using the thermal neutron tripleaxis spectrometer-Taipan in the same scattering plane as on Pelican. The scan directions are summarized in Supplementary Fig. 7. Figure $3 c$, d shows the dispersions along $[H, 0,0]$ and $[0, L, L]$ directions of Brillouin zone $\mathbf{G}=(200)$ at $300 \mathrm{~K}$ obtained by constant- $E$ scans, respectively. Note that while Fig. $3 c$ represents the longitudinal component of $\mathbf{G}=(200)$ Brillouin zone, some contributions of the longitudinal phonons are also included in Fig. $3 \mathrm{~d}$ in addition to the major transverse components (see Supplementary Fig. 7). The phonon intensity decays quickly with departure from the Brillouin zone center. To accurately determine the dispersion relationships, the phonon energies are determined by a spectral fitting to a Lorentzian function. The obtained peak positions are plotted on the contour plots as circles with error bars. Then, we fit the phonon energies to a linear function approaching the zone centers to obtain the experimental sound speeds (see the dash lines). The derived values are listed in Supplementary Table 4 . It can be seen that the dispersion along $[H, 0,0]$ direction is much steeper than that along $[2, L, L]$ direction, indicating larger sound speeds. A similar procedure is applied to the cases of $\mathbf{G}=(022)$ and $\mathbf{G}=(111)$, as shown in Fig. $3 \mathrm{e}-\mathrm{h}$, respectively.

Lattice anharmonicity. The temperature-dependent lattice dynamics are considered first on the phonon density of state (PDOS) measurements on a powder sample in a wide temperature region. Shown in Fig. 4a are PDOS at 200, 300, 400, 500, and $600 \mathrm{~K}$, respectively. As temperature rises, it is clear that the whole profile is significantly broadened and several peaks are remarkably softened as an indication of strong anharmonicity. Compared with the DFT calculated PDOS, as plotted in Fig. $4 \mathrm{~b}$, it is identified that the $\mathrm{Cu}$ atoms mainly participate in the low-energy 
a

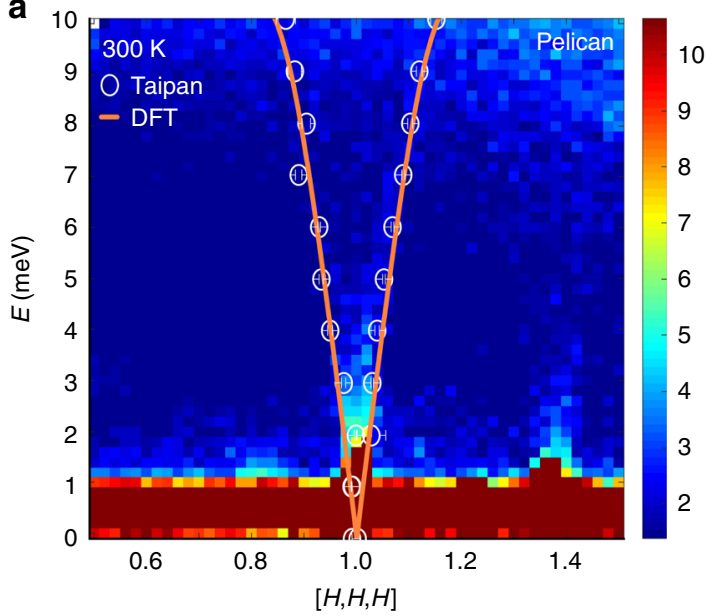

b 16

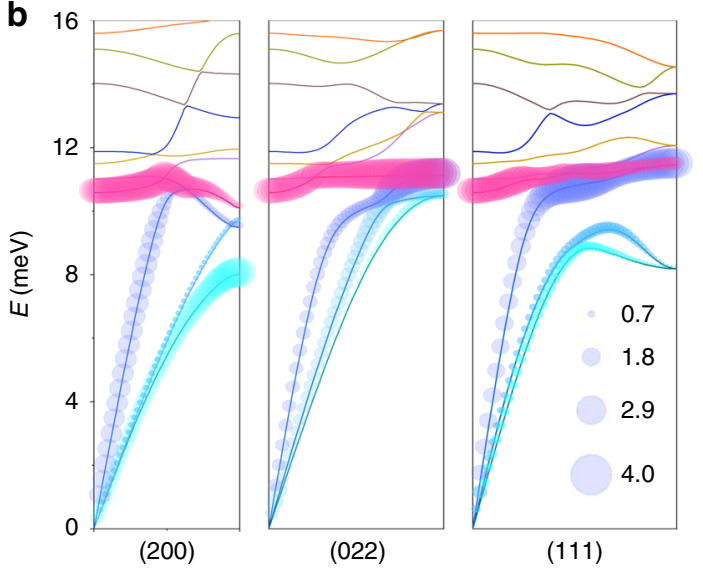

c
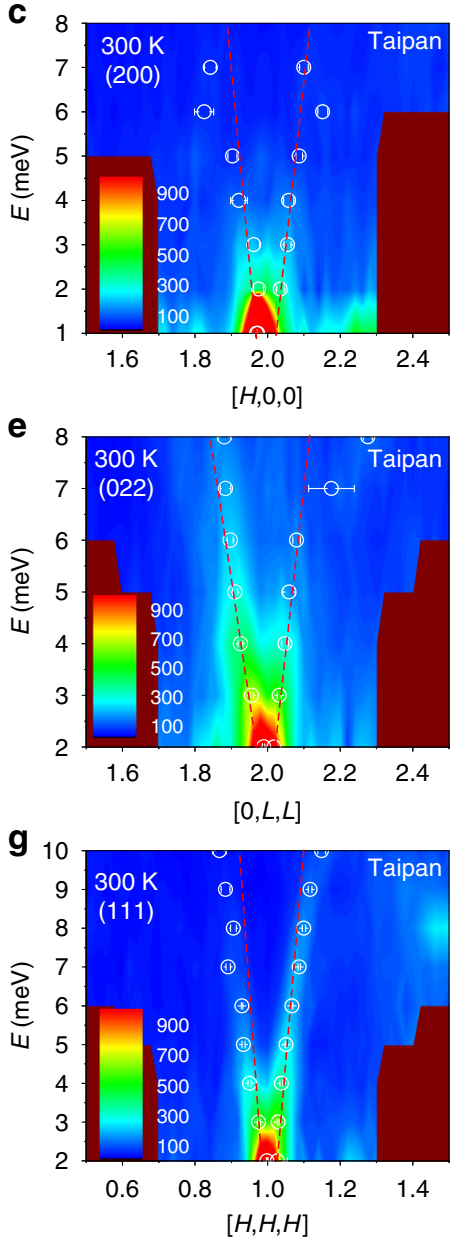

d 10

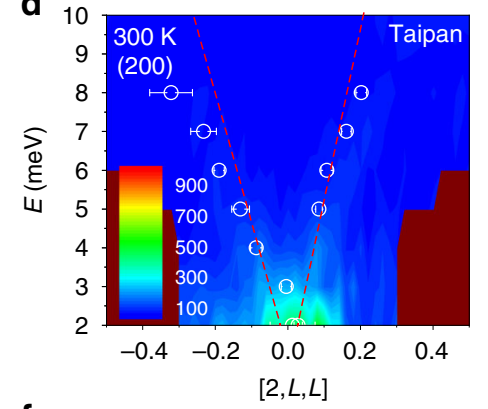

$\mathbf{f}$

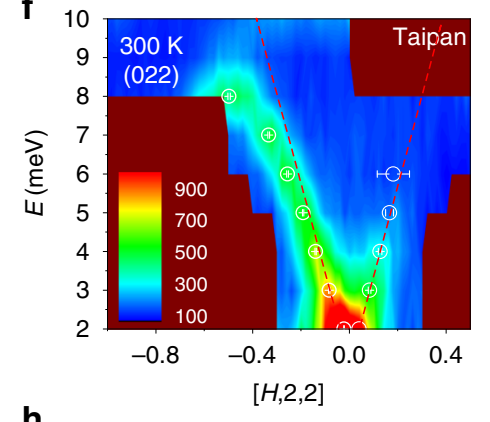

h 10

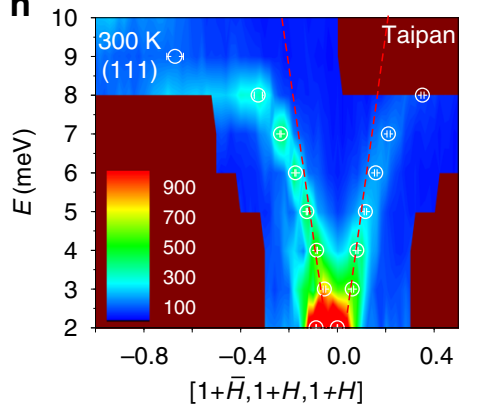

Fig. 3 Phonon dispersions of $\mathbf{C u P}_{\mathbf{2}}$. a The phonon dispersion obtained at the time-of-flight neutron spectrometer-Pelican along $[H, H, H]$ direction of

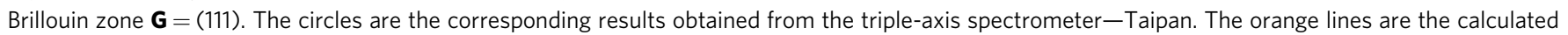
dispersions. b Calculated phonon dispersions of Brillouin zones $\mathbf{G}=(200), \mathbf{G}=(022)$, and $\mathbf{G}=(111)$. The size of the shadow bubbles represents the

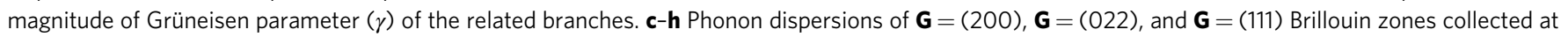

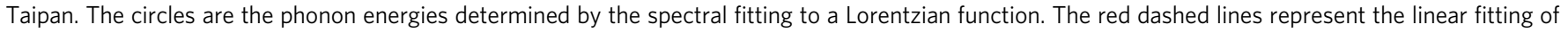
phonon branches approaching the Brillouin zone centers to determine the sound speeds.

modes below $15 \mathrm{meV}$, while the high-energy optical modes are dominated by $\mathrm{P}$ atoms. This might be related to the fact that the covalent interactions of $\mathrm{P}$ networks are much stronger, in addition to the lighter atomic mass of $\mathrm{P}$ than that of the $\mathrm{Cu}$ dimer. Of particular interest is the experimentally observed mode located at about $11 \mathrm{meV}$. Compared with the DFT calculations of the phonon dispersion (Fig. 3b) and PDOS (Fig. 4b), this mode is associated with $\mathrm{Cu}$ and has a flat dispersion, thus it must be a rattling mode contributed by the $\mathrm{Cu}$ dimer (justified later). The strong anharmonicity of this rattling mode is demonstrated by the large softening from $11.40 \mathrm{meV}$ at $200 \mathrm{~K}$ to $10.67 \mathrm{meV}$ at $600 \mathrm{~K}$. Since the acoustic phonons and the nearby low-frequency optical phonons are the main contributors to the thermal conductivity, we further investigate the anharmonic property of this $\mathrm{Cu}$ rattling mode in the following sections.

Focusing on the significantly softened mode at about $11 \mathrm{meV}$, detailed constant-Q scans were performed at 100,300 , and $450 \mathrm{~K}$, respectively. Figure $4 \mathrm{c}$ shows the dispersion along $[H, 0,0]$ direction of $\mathbf{G}=(022)$ at $100 \mathrm{~K}$ (the results at 300 and $450 \mathrm{~K}$ are shown in Supplementary Fig. 8). Just above the intense acoustic phonon branch, a very flat optical phonon branch originates from the Brillouin zone center at about $12 \mathrm{meV}$ to the Brillouin zone boundary at about $10 \mathrm{meV}$. This is the rattling mode of the $\mathrm{Cu}$ dimer. At higher energies, three more flat branches are further observed. The temperature dependencies of these modes are well tracked at the constant- $\mathbf{Q}$ scans at $\mathbf{q}=0,0.2,0.3$, as shown in Fig. $4 \mathrm{~d}-\mathrm{f}$, respectively. Data at $\mathbf{q}=0$ clearly indicate a distinct behavior for the three optical modes identified. The energy of the first mode (rattling mode) is reduced from $11.16 \mathrm{meV}$ at $100 \mathrm{~K}$ to $10.24 \mathrm{meV}$ at $450 \mathrm{~K}$, at a similar rate of $-0.002 \mathrm{meV} \mathrm{K}^{-1}$ as observed with the PDOS results. In contrast, the other two modes at 14.53 and $15.53 \mathrm{meV}$ at $100 \mathrm{~K}$ hardly shift with increasing temperature. At $\mathbf{q}=0.2$ and 0.3 shown in Fig. $4 \mathrm{e}, \mathrm{f}$, it is further noticed that the acoustic mode around $6 \mathrm{meV}$ shows no noticeable shift in energy upon temperature increase in contrast to the rattling mode. This behavior is also shown on more data as presented in Supplementary Figs. 9 and 10 for the Brillouin zone $\mathbf{G}=(022)$. This feature is in sharp contrast to most materials, such as $\mathrm{FeSi}^{26}$, where phonon modes are not selectively softened with increasing temperature.

Apart from the remarkable temperature-induced softening of the rattling mode as discussed above, the strong anharmonicity is also manifested by the huge Grüneisen parameter $(\gamma)^{27,28}$ calculated by DFT. In Fig. 3b, the size of the shadow bubbles represents the magnitude of $\gamma$ of a specific mode. Both the rattling mode and the acoustic modes have very large $\gamma$. The 

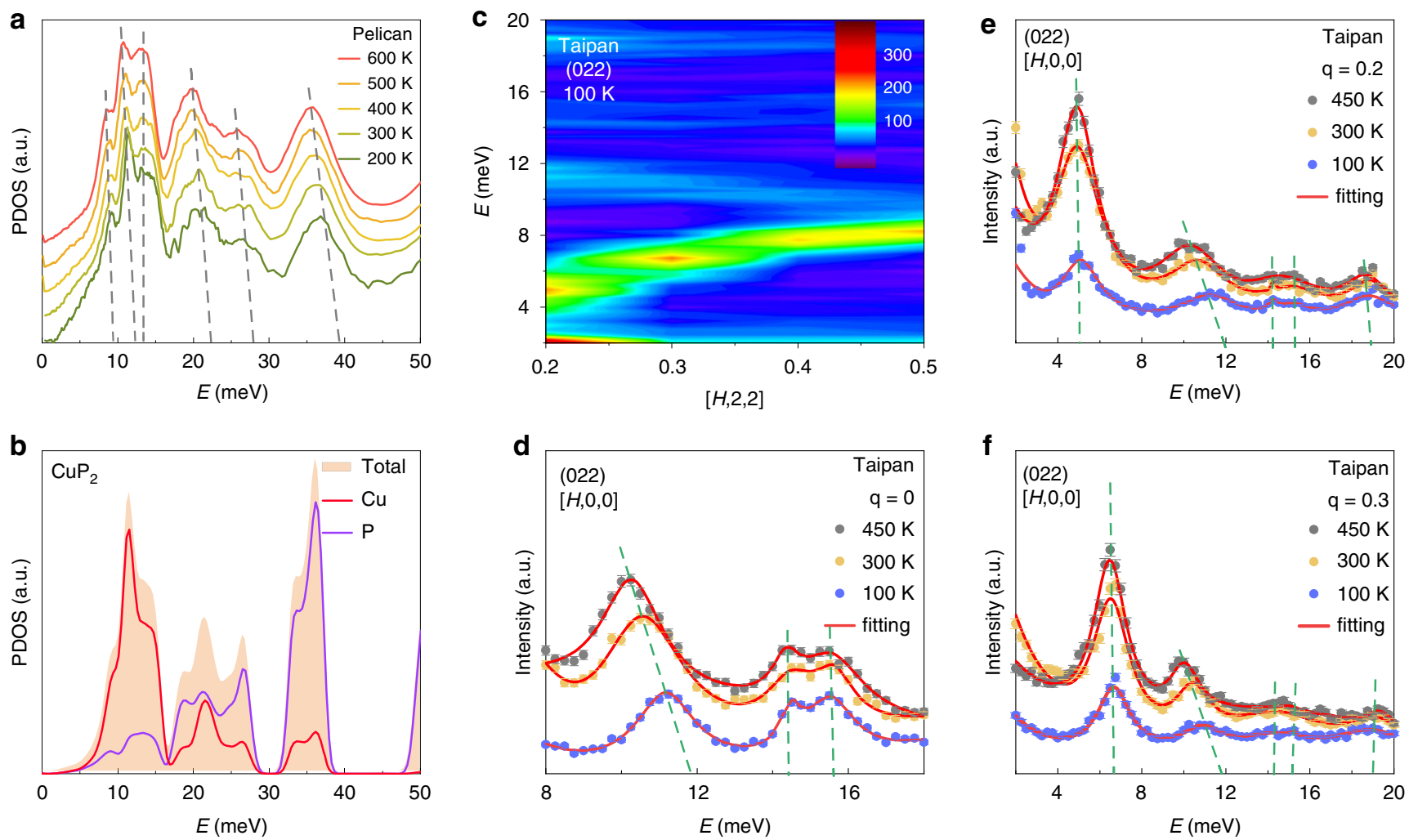

Fig. 4 Highly anharmonic optical mode at 11 meV. a The experimental PDOS, vertically shifted for clarity. b. Calculated total and partial PDOS. c. Phonon dispersion of $\mathbf{G}=(022)$ Brillouin zone along $[H, 0,0]$ at $100 \mathrm{~K}$. d-f Temperature dependence of phonon spectra for constant- $\mathbf{Q}$ scans of $\mathbf{G}=(022)$ Brillouin zone along $[\mathrm{H}, \mathrm{O}, 0]$ with $\mathbf{q}=0,0.2,0.3$ at 100,300 and $450 \mathrm{~K}$, respectively. The green dash lines highlight the energy shift of the peaks at heating.

\begin{tabular}{|c|c|c|c|}
\hline \multirow[t]{2}{*}{$U\left(\AA^{3}\right)$} & \multirow{2}{*}{$\begin{array}{l}\mathrm{Cu} \\
\mathrm{Cu} 1\end{array}$} & \multicolumn{2}{|l|}{$\mathbf{P}$} \\
\hline & & P1 & P2 \\
\hline$U_{111}$ & $0.0006(4)$ & $0.0001(7)$ & $0.0009(13)$ \\
\hline$U_{222}$ & $0.0025(6)$ & $0.0011(9)$ & $0.00021(20)$ \\
\hline$U_{333}$ & $0.00090(13)$ & $0.00021(20)$ & $-0.0001(2)$ \\
\hline
\end{tabular}

largest $\gamma$ for the rattling mode is about 3.3 at $\mathbf{q}=0.5$, corresponding to the $\mathbf{G}=(022)$ Brillouin zone boundary, while $\gamma$ is almost larger than 2 in all reciprocal space covered. In particular, $\gamma$ is strikingly large for the longitudinal acoustic (LA) branches (blue color) near the zone boundaries, where they tend to overlap with the rattling mode. In contrast, the Grüneisen parameter of GaAs is almost less than 1 in the whole temperature region below $300 \mathrm{~K}$ (ref. ${ }^{29}$ ). As a result, the anharmonicity of $\mathrm{CuP}_{2}$ is much stronger than that of $\mathrm{GaAs}$ with the same magnitude of sound speed, resulting in the huge difference in the lattice thermal conductivity between the two materials. Moreover, our single-crystal X-ray diffraction measurement also suggests that $\mathrm{Cu}$ atoms have substantial third-order Debye-Waller factors, which provides further evidence of the strong anharmonicity of $\mathrm{Cu}$-involved vibrations, as listed in Table 1 . The values of $U_{222}$ and $U_{333}$ are $0.0025(6)$ and $0.00090(13) \AA^{3}$ while $U_{111}$ is as small as 0.0006 (4) $\AA^{3}$. Such a difference is similar to the second-order Debye-Waller factors plotted in Supplementary Fig. 4d. As compared to $\mathrm{Cu}$, the third-order Debye-Waller factors of $\mathrm{P}$ atoms are almost negligible within the error bars.

Rattling mode and thermal conductivity. Henceforward, we discuss the nature of the highly anharmonic rattling mode at 11 $\mathrm{meV}$ and its impact on the system. Atomic motions of this mode are plotted at the $\Gamma$ point, as shown in Fig. $1 \mathrm{c}$, d, which involve the vibrations of $\mathrm{Cu}$ dimers in between the $\mathrm{P}$ layers. The atomic motions are recorded in Supplementary Movie 1 and those of the nearby two modes are also present in Supplementary Movie 2 and Supplementary Movie 3, respectively. The justification of calling the vibration of the $\mathrm{Cu}$ dimers a rattling mode is further articulated here. Firstly, the $\mathrm{Cu}$ dimers behaving as rattlers have very weak bonding to the others as crystallographically and electronically confirmed above. This is also evidenced by the significant Debye-Waller factors of $\mathrm{Cu}$ atoms that represent large-amplitude anharmonic vibrations in $b c$ plane, in agreement with the well-known skutterudites and clathrates compounds ${ }^{10,30,31}$. Secondly, the dispersion of this mode is quite flat as the group velocity is smaller than $10 \mathrm{~m} \mathrm{~s}^{-1}$ showing an uncorrelated and localized feature. This is in close analogy to the ideal rattling scenario, appearing as a dispersionless Einstein mode with a constant vibration frequency. Thirdly, the anti-crossing or avoided-crossing of the acoustic mode with the rattling mode is also theoretically predicted in the compound, though not experimentally observed due to experiment limitations, as highlighted in Supplementary Fig. 6. The theory predicted five anti-crossing points located at $\mathbf{q}=$ 0.27 of $\mathbf{G}=\left(1 / 2^{1 / 2} 0\right), \quad \mathbf{q}=0.32$ of $\mathbf{G}=(1 / 200), \quad \mathbf{q}=0.37$ of $\mathbf{G}=(001 / 2), \quad \mathbf{q}=0.13$ of $\mathbf{G}=(\overline{1 / 2} 1 / 20), \quad$ and $\quad \mathbf{q}=0.32$ of $\mathbf{G}=(\overline{1 / 2} 00)$. This behavior also gives rise to the decrease of the 
group velocity of the acoustic mode around the zone boundaries. This rattling mode differs from the previous ones reported in skutterudites and clathrates systems. These compounds have typical cage-like frames with the heavy atoms filled into the lattice void, whereas $\mathrm{CuP}_{2}$ crystallizes in a layered structure with $\mathrm{Cu}$ dimer as the rattler ${ }^{10-13,32}$. To distinguish with the conventional rattling mode, we term the behavior of $\mathrm{CuP}_{2}$ as dimer rattling.

This rattling mode is expected to play a dominating role in the thermal transport. We have systematically determined the sound speeds both experimentally and theoretically, as summarized in Supplementary Table 4. For example, $v_{\mathrm{LA}}=6243 \mathrm{~m} \mathrm{~s}^{-1}$ and $v_{\mathrm{TA}}=3192 \mathrm{~m} \mathrm{~s}^{-1}$ are obtained from DFT calculations for the LA and transverse acoustic (TA) branches of $\mathbf{G}=(200)$, respectively. This $v_{\mathrm{LA}}$ value is in excellent agreement with that determined by both fitting the INS experimental phonon dispersions and the Brillouin light scattering spectrum (Supplementary Fig. 11), which are $6364 \mathrm{~m} \mathrm{~s}^{-1}$ and $6275 \mathrm{~m} \mathrm{~s}^{-1}$, respectively. The mean sound speed $v_{\mathrm{m}}=4155 \mathrm{~m} \mathrm{~s}^{-1}$ is estimated using the equation $3 v_{\mathrm{m}}^{-3}=$ $v_{\mathrm{LA}}^{-3}+2 v_{\mathrm{TA}}^{-3}$ (ref. ${ }^{13}$ ) based on the theoretical results. As shown in Fig. 1d, unlike the ordinary materials that are distributed around the empirical line, $\mathrm{CuP}_{2}$ stands quite exceptional with the thermal conductivity one order of magnitude lower than GaAs that has a similar mean sound speed as $\mathrm{CuP}_{2}^{22,23}$.

\section{Discussion}

We have discovered the suppressed thermal transport property of $\mathrm{CuP}_{2}$ and established a profound understanding on the fundamental physical mechanism by a comprehensive study on the atomic structures and lattice dynamics through neutron, $\mathrm{X}$ ray scattering techniques, and complementary DFT calculations. This system is manifested to be very anharmonic. The $\mathrm{Cu}$ atoms participate in a dimer rattling mode, which strongly scatters the LA phonons and leads to anti-crossing phenomena in the dispersion relationships. It is this mode that dominates the reduced thermal conductivity, counteracting the contribution of large mean sound speeds. The observed dimer rattling behavior in the open layered structures might represent an emerging opportunity to rationally tailor thermal transport properties of solids. The combined excellent acoustic conduction and thermal insulation properties may find $\mathrm{CuP}_{2}$ a promising material in some nontrivial applications requiring both excellent mechanical sound transmission (or mechanical rigidity) and heat insulation.

\begin{abstract}
Methods
Sample preparation. $\mathrm{CuP}_{2}$ single crystals were grown through a flux method ${ }^{33}$. Starting materials of Cu (purity: 99.999\%), P (purity: 99.999\%), and Sn (purity: $99.999 \%$ ) in a molar ratio of 1:1:3 were placed in an alumina crucible and then sealed into an evacuated quartz tube. The mixture was placed into a box furnace and heated at $1233 \mathrm{~K}$ for $6 \mathrm{~h}$. Then, it was cooled down to $873 \mathrm{~K}$ at a rate of $3 \mathrm{~K} / \mathrm{h}$. The excessive $\mathrm{Cu}$ and $\mathrm{Sn}$ eutectic flux were decanted in a centrifuge at $873 \mathrm{~K}$. Single crystals were mechanically cleaved from the ingots. Single crystals were crushed for neutron and X-ray powder diffraction as well as for thermal conductivity measurement where a carbon-coated pellet $(\phi=10 \mathrm{~mm})$
\end{abstract} was used.

Thermal conductivity measurement. The total thermal conductivity $\left(\kappa_{\mathrm{tot}}\right)$ of the polycrystalline sample was obtained based on the formula $\kappa_{\text {tot }}=D C_{\mathrm{p}} \rho$, where $D$ is the thermal diffusion coefficient measured using the laser flash method (LFA457, NETZSCH, Germany), $C_{\mathrm{p}}$ is the Dulong-Petit specific heat capacity, and $\rho$ is the density calculated from the geometrical dimensions and mass. Thermal conductivity of single crystals was measured in the temperature range from about 50 to $300 \mathrm{~K}$ using a steady-state comparative method ${ }^{34}$. The bar-shaped $\mathrm{CuP}_{2}$ samples with a dimension of about $0.5 \times 0.5 \times 3 \mathrm{~mm}^{3}$ were cut from as-grown crystals. The reference was a rod of constantan alloy with a diameter of $0.5 \mathrm{~mm}$. The differential thermocouple was made of copper and constantan wires. In this method, the thermal conductivity of the sample is obtained by measuring the temperature difference between the heat source and heat sink under a steady heat flow through the bar-shaped sample (consist of $\mathrm{CuP}_{2}$ single crystal and the reference). The uncertainty of the steady state comparative method is related to the error in measuring sample dimensions, contact thermal resistance, and heat loss. The uncertainty is usually about $10-20 \%$ (ref. ${ }^{35}$ ). The total thermal conductivity is expressed as a sum of lattice contribution $\left(\kappa_{\text {lat }}\right)$ and electronic contribution $\left(\kappa_{\mathrm{el}}\right)$ for this compound

$$
\kappa_{\text {tot }}=\kappa_{\text {lat }}+\kappa_{\mathrm{el}} .
$$

The electronic part $\kappa_{\mathrm{el}}$ is proportional to the electrical conductivity $\sigma$ through the Wiedemann-Franz relation

$$
\kappa_{\mathrm{el}}=L \sigma T,
$$

where $L$ is Lorenz number ${ }^{36}$. The lattice thermal conductivity $\kappa_{\text {lat }}$ can be estimated by subtracting the electrical contribution $\kappa_{\mathrm{e}}$. The electrical conductivity of $\mathrm{CuP}_{2}$ is only about $8.09 \Omega^{-1} \mathrm{~cm}^{-1}$ at $300 \mathrm{~K}$ (ref. ${ }^{37}$ ). $\kappa_{\mathrm{el}}$ of $\mathrm{CuP}_{2}$ is about $0.0059 \mathrm{~W} \mathrm{~m}^{-1} \mathrm{~K}^{-1}$ by assuming $L=2.45 \mathrm{~W} \Omega \mathrm{K}^{-2}$ (ref. ${ }^{38}$ ). In general, the true Lorenz number $L$ for most thermoelectric materials is lower than this value so that the electronic contribution to the thermal conductivity is negligible in this system ${ }^{39}$.

Neutron powder diffraction. The neutron powder diffraction was performed at the time-of-flight powder diffractometer at the Spallation Neutron Source of Oak Ridge National Laboratory, USA ${ }^{40}$. The powder sample with the mass around $3 \mathrm{~g}$ was loaded into a vanadium container of $8 \mathrm{~mm}$ diameter and measured in a Powgen Automatic Changer covering the temperature region of $10-300 \mathrm{~K}$. The data were collected with neutrons of central wavelength $0.8 \AA$. Constant temperature scans were conducted at $10,50,100,200$, and $300 \mathrm{~K}$, respectively. GSAS ${ }^{41}$ was used to refine the neutron powder diffraction patterns and the results are summarized in Fig. 2a, Supplementary Figs. 3 and 4 as well as Table 3. The Rietveld method employs a nonlinear least-square method to fit the profile that includes all structural and instrumental parameters ${ }^{42}$. Several figure-of-merits including $R_{\mathrm{p}}$, $R_{\mathrm{wp}}$, and $\chi^{2}$ that mean profile residual (reliability factor), weighted profile residual, and goodness of fit are used to quantify the quality of the fit.

Synchrotron X-ray total scattering. The high-energy X-ray powder diffraction experiment was carried out at the Beamline BL04B2 of SPring-8, Japan ${ }^{43}$. The $\mathrm{CuP}_{2}$ powder was put into a quartz capillary $(\phi=1 \mathrm{~mm})$ and then fixed into the sample holder. An empty capillary was measured as a background. X-ray energy was fixed at $61.4 \mathrm{keV}$ with a $\mathrm{Si}(220)$ monochromator. The energy resolutions $\Delta E / E$ was approximately $5 \times 10^{-3}$. A vacuum chamber with a Kapton window was used for minimizing the scattering background. Six point-detectors were arranged horizontally to obtain $2 \theta$ value up to $49^{\circ}\left(Q\right.$ range up to $\left.25 \AA^{-1}\right)$. The real-space refinement was performed using PDFgui ${ }^{44}$.

Single-crystal X-ray diffraction analysis. The single-crystal X-ray diffraction data were collected at a Pilatus CCD diffractometer equipped with graphitemonochromated $\mathrm{Mo}-K_{\alpha}$ radiation $(\lambda=0.71073 \AA)$ at $293 \mathrm{~K}$. The crystal structure of $\mathrm{CuP}_{2}$ was solved, and three-order atomic thermal displacement factors of all atoms were refined through full-matrix least-square technique on $F^{2}$. All of the calculations were performed using Jana2006 (ref. ${ }^{45}$ ).

INS measurements. INS experiments were first performed on the time-of-flight cold-neutron spectrometer-Pelican ${ }^{46,47}$, at the Australian Nuclear Science and Technology Organization (ANSTO), Australia. For the PDOS measurements, a powder sample was mounted in an annular aluminum sample can with $1 \mathrm{~mm}$ gap. The sample can was attached to the cold head of a closed-cycle refrigerator which is capable of achieving sample temperature from 1.5 to $800 \mathrm{~K}$. The instrument was aligned for $4.69 \AA(3.7 \mathrm{meV})$ incident neutrons. The resolution at the elastic line was $135 \mu \mathrm{eV}$. The intensity of an empty can was subtracted as the background contribution and the data were normalized to a vanadium standard that had the same geometry as the sample can. All data manipulations were performed using the Large Array Manipulation Program (LAMP $)^{48}$. The scattering function $S(\mathbf{Q}, E)$, as a function of scattering wave vectors $(\mathbf{Q})$ and phonon energy $(E)$, were measured on energy gain mode over a wide temperature range and then transformed to a generalized PDOS using formula (3), where $k_{B}$ is Boltzmann's constant and $T$ is temperature

$$
g(E)=\int \frac{E}{Q^{2}} S(Q, E)\left(1-e^{-\frac{E}{k_{B} T}}\right) d Q .
$$

For phonon dispersion measurements, a single crystal weighted $0.7 \mathrm{~g}$ was used and pre-orientated such that the scattering plane was defined by $[H, 0,0]$ and $[0, L, L]$. A large reciprocal space was covered by rotating the sample over $100^{\circ}$ in a step of $1^{\circ}$ around the axis perpendicular to the scattering plane. The incident neutron wavelength is $2.345 \AA(14.8 \mathrm{meV})$, corresponding to the second-order reflection of the Pelican highly oriented pyrolytic graphite monochromators configured at $4.69 \AA$. The sample temperature was set at $300 \mathrm{~K}$. The data were reduced by LAMP and the whole $S(\mathbf{Q}, E)$ was generated using HORACE ${ }^{49}$, which was also used to visualize the phonon dispersions. After the general survey over many Brillouin zones using the Pelican instrument, we focused on several selected Brillouin zones $\mathbf{G}=(200), \mathbf{G}=(022)$, and $\mathbf{G}=(111)$ on the thermal neutron triple- 
axis spectrometer-Taipan at ANSTO $^{50,51}$. The energies of the incident and scattered neutrons were defined by a double-focused PG (002) monochromator and analyzer. We used the open geometry of the neutron beam with a virtual source width of $10 \mathrm{~mm}$ at the beam exit from the reactor and a slit of $20 \mathrm{~mm}$ at the detector. The measurements were performed with fixed final neutron energy of $14.87 \mathrm{meV}$. After the sample, a HOPG filter was placed to remove higher-order reflections from the scattered beam. The sample was aligned in the same scattering plane as in the measurements at Pelican. The phonons were measured along $[H, 0,0],[H, H, H]$, and $[0, L, L]$ directions. A standard cryofurnace was used to access the temperatures of 100,300 , and $450 \mathrm{~K}$. The experimental data were fitted to a Lorentzian function using PAN of DAVE ${ }^{52}$

Brillouin light scattering. Brillouin light scattering measurements were performed at room temperature using a Sandercock-type six-pass tandem Fabre-Perot (TFP2) as a spectrometer and a $532 \mathrm{~nm}$ laser as a light source. It is a well-established technique for measuring sound velocities ${ }^{53}$. In backscattering geometry, LA mode sound velocity $\left(v_{\mathrm{LA}}\right)$ can be calculated as

$$
v_{\mathrm{LA}}=\frac{f_{\mathrm{B}} \lambda_{0}}{2 n} .
$$

From the measured Brillouin frequency shift $f_{\mathrm{B}}$, using this equation, the refractive index $n=4.04$ and the laser wavelength $\lambda_{0}=532 \mathrm{~nm}$, the LA-wave velocity was determined to be $v_{\mathrm{LA}}=6275 \mathrm{~m} \mathrm{~s}^{-1}$.

DFT calculations. All the calculations in this work were performed using the ab initio DFT as implemented in the VASP $\operatorname{code}^{54}$. The projector augmented wave pseudopotentials ${ }^{55}$ and the Perdew-Burke-Ernzerhof ${ }^{56}$ functional within the general gradient approximation were used to take care of electron-ion and inter-electron exchange-correlation interactions, respectively. The wave functions were expanded using plane waves with an energy cutoff of $500 \mathrm{eV}$, and the electronic energy convergence was set to be $10^{-8} \mathrm{eV}$. The Brillouin zone of the primitive unit cell was sampled in the $\Gamma$-centered $8 \times 10 \times 15 k$-point mesh for structural optimization until all the atomic force is less than $0.001 \mathrm{eV}^{-1}$. The phonon dispersion relation was calculated using Phonopy package ${ }^{57}$ combining with VASP using $2 \times 2 \times 2$ supercell with 96 atoms. The $\Gamma$-centered $2 \times 2 \times 2 q$-point mesh was used. To unravel the chemical bonding nature of the $\mathrm{Cu}-\mathrm{Cu}$ atomic pair, we also calculated the ELF ${ }^{58}$ and electronic charge transfer $\Delta \rho=\rho\left(\mathrm{CuP}_{2}\right)-\rho$ (atom), which is defined as the electron charge density difference between $\mathrm{CuP}_{2}$ and the constituent individual atoms.

\section{Data availability}

Available from the corresponding authors on reasonable request.

Received: 9 June 2020; Accepted: 28 September 2020;

Published online: 15 October 2020

\section{References}

1. Tritt, T. M. Thermal Conductivity: Theory, Properties, and Applications (Klumer Academic/Plenum Publishers, New York, USA, 2004).

2. Padture, N. P., Gell, M. \& Jordan, E. H. Thermal barrier coatings for gasturbine engine applications. Science 296, 280-284 (2002).

3. Siegrist, T., Merkelbach, P. \& Wuttig, M. Phase change materials: challenges on the path to a universal storage device. Annu. Rev. Condens. Matter Phys. 3, 215-237 (2012).

4. Bell, L. E. Cooling, heating, generating power, and recovering waste heat with thermoelectric systems. Science 321, 1457-1461 (2008).

5. Holland, M. G. Analysis of lattice thermal conductivity. Phys. Rev. 132, 2461-2471 (1963).

6. Callaway, J. Model for lattice thermal conductivity at low temperatures. Phys. Rev. 113, 1046-1051 (1959).

7. Walliams, R. K. et al. Experimental and theoretical evaluation of the phonon thermal conductivity of niobium at intermediate temperatures. Phys. Rev. B 28, 6316-6324 (1983).

8. Delaire, O. et al. Giant anharmonic phonon scattering in PbTe. Nat. Mater. 10, 614-619 (2011)

9. Li, C. W. et al. Orbitally driven giant phonon anharmonicity in SnSe. Nat. Phys. 11, 1064-1070 (2015).

10. Sales, B. C. et al. Filled skutterudite antimonides: electron crystals and phonon glasses. Phys. Rev. B 56, 15081-15089 (1997)

11. Keppens, V. et al. Localized vibrational modes in metallic solids. Nature 395, 876-878 (1998).

12. Slack, G. A. CRC Handbook of Thermoelectrics (CRC Press, Boca Raton, USA, 1995).

13. Christensen, M. et al. Avoided crossing of rattler modes in thermoelectric materials. Nat. Mater. 7, 811-815 (2008).
14. Zhang, Q. Y. et al. Enhencement of thermoelectric figure-of-merit by resonant states of aluminum doping in lead selenide. Energy Environ. Sci. 5, 5246-5251 (2012).

15. Minnich, A. J., Dresselhaus, M. S., Ren, Z. F. \& Chen, G. Bulk nanostructured thermoelectric materials: current research and future prospects. Energy Environ. Sci. 2, 466-479 (2009)

16. Poudel, B. et al. High-thermoelectric performance of nanostructured bismuth antimony telluride bulk alloys. Science 320, 634-638 (2008).

17. Liu, H. et al. Copper ion liquid-like thermoelectrics. Nat. Mater. 11, 422-425 (2012).

18. $\mathrm{Li}, \mathrm{B}$. et al. Liquid-like thermal conduction in intercalated layered crystalline solid. Nat. Mater. 17, 226-230 (2018).

19. $\mathrm{Li}, \mathrm{W}$. et al. Low sound velocity contributing to the high thermoelectric performance of $\mathrm{Ag}_{8} \mathrm{SnSe}_{6}$. Adv. Sci. 3, 1600196 (2016).

20. Slack, G. A. Thermal conductivity of pure and impure silicon, silicon carbide, and diamond. J. Appl. Phys. 35, 3460-3466 (1964).

21. Inyushkin, A. V. \& Taldenkov, A. N. Thermal conductivity of high purity synthetic single crystal diamonds. Phys. Rev. B 97, 144305 (2018).

22. Madelung, O. Semiconductors: Data Handbook (Springer Science \& Business Media, Marburg, Germany, 2012).

23. Nakwaski, W. O. Thermal conductivity of binary, ternary, and quaternary IIIV compounds. J. Appl. Phys. 64, 159-166 (1988).

24. Rowe, D. M. \& Shukla, V. S. The effect of phonon-grain boundary scattering on the lattice thermal conductivity and thermoelectric conversion efficiency of heavily doped fine-grained, hot-pressed silicon germanium alloy. J. Appl. Phys. 52, 7421-7426 (1981).

25. Olofsson, $\mathrm{O}$. The crystal structure of $\mathrm{CuP}_{2}$ and $\mathrm{AgP}_{2}$ with some phase analytical data of the Cu-P and Ag-P systems. Acta Chem. Scand. 19, 229-241 (1965).

26. Delaire, O. et al. Effects of temperature and pressure on phonons in $\mathrm{FeSi}_{1-\mathrm{x}} \mathrm{Al}_{\mathrm{x}}$. Phys. Rev. B 87, 184304 (2013).

27. Lin, $\mathrm{H}$. et al. Concerted rattling in $\mathrm{CsAg}_{5} \mathrm{Te}_{3}$ leading to ultralow thermal conductivity and high thermoelectric performance. Angew. Chem. Int. Ed. 55, 11431-11436 (2016).

28. Xiao, Y. et al. Origin of low thermal conductivity of SnSe. Phys. Rev. B 94, 125203 (2016).

29. Cottam, R. I. \& Saunders, G. A. The elastic constants of GaAs from 2 K to 320 K. J. Phys. C 6, 2105-2118 (1973).

30. Koza, M. M. et al. Breakdown of phonon glass paradigm in La- and Ce-filled $\mathrm{Fe}_{4} \mathrm{Sb}_{12}$ skutterudites. Nat. Mater. 7, 805-810 (2008).

31. Sales, B. C. et al. Structural, magnetic, thermal, and transport properties of $\mathrm{X}_{8} \mathrm{Ga}_{16} \mathrm{Ge}_{30}(\mathrm{X}=\mathrm{Eu}, \mathrm{Sr}, \mathrm{Ba})$ single crystals. Phys. Rev. B 63, 245113 (2001).

32. Samanta, M. et al. Localized vibrations of Bi bilayer leading to ultralow lattice thermal conductivity and high thermoelectric performance in weak topological insulator $n$-type BiSe. J. Am. Chem. Soc. 140, 5866-5872 (2018).

33. Kloc, C. et al. Growth and characterization of $\mathrm{CuP}_{2}$ single crystals. J. Cryst. Growth 106, 635-642 (1990).

34. Chen, $X$. et al. Effects of grain boundaries and defects on anisotropic magnon transport in textured $\mathrm{Sr}_{14} \mathrm{Cu}_{24} \mathrm{O}_{41}$. Phys. Rev. B 95, 144310 (2017).

35. Tian, F. et al. Unusual high thermal conductivity in boron arsenide bulk crystals. Science 361, 582-585 (2018).

36. Fitsul, V. I. Heavily Doped Semiconductors (Plenum Press: New York, USA, 1969).

37. Goryunova, N. A. et al. Preparation and some properties of $\mathrm{CuP}_{2}$ single crystals. Phys. Stat. Sol. 25, 513-519 (1968).

38. Sommerfeld, A. Zur elektronentheorie der metalle. Die Naturwissenschaften 41, 825-832 (1927).

39. Kumar, G. S., Prasad, G. \& Pohl, R. O. Experimental determinations of the Lorenz number. J. Mater. Sci. 28, 4261-4272 (1993).

40. Huq, A., Hodfes, J. P. \& Gourdon, O. Powgen: a third-generation highresolution high-throughput powder diffraction instrument at the spallation neutron source. Z. Kristallogr. Proc. 1, 127-135 (2011).

41. Toby, B. H. EXPGUI, a graphical user interface for GSAS. J. Appl. Cryst. 34, 210-213 (2001).

42. Rietveld, H. M. A profile refinement method for nuclear and magnetic structures. J. Appl. Cryst. 2, 65-71 (1969).

43. Isshiki, M., Ohishi, Y., Goto, S., Takeshita, K. \& Ishikawa, T. High-energy Xray diffraction beamline: BL04B2 at SPring-8. Nucl. Instr. Methods Phys. Res. A 467-468, 663-666 (2001).

44. Farrow, C. L. PDFfit2 and PDFgui: computer program for studying nanostructure in crystals. J. Phys. Condens. Matt. 19, 335219 (2007).

45. Petř́čck, V., Dušek, M. \& Palatinus, L. Crystallographic computing system JANA2006: General features. Z. Kristallogr. 229, 345-352 (2014).

46. $\mathrm{Yu}, \mathrm{D}$. et al. Performance test on PELICAN-a multi-purpose time of flight cold neutron spectrometer. EPJ Web Conf. 83, 03019 (2015).

47. Yu, D. et al. Pelican-a time of flight cold neutron polarization analysis spectrometer at OPAL. J. Phys. Soc. Jpn. 82, SA027 (2013).

48. Richard, D. et al. Analysis and visualisation of neutron-scattering data. J. Neutron Res. 4, 33-39 (1996). 
49. Ewings, R. A. et al. HORACE: Software for the analysis of data from single crystal spectroscopy experiments at time-of-flight neutron instruments. Nucl. Inst. Methods Phys. Res. A 834, 132-142 (2016).

50. Danilkin, S. A. et al. The TAIPAN thermal triple-axis spectrometer at the OPAL reactor. J. Neutron Res. 15, 55-60 (2007).

51. Danilkin, S. A. \& Yethiraj, M. TAIPAN: thermal triple-axis spectrometer. Neutron News 20, 37-39 (2009).

52. Azuah, R. T. et al. DAVE: a comprehensive software suite for the reduction, visualization, and analysis of low energy neutron spectroscopic data. J. Res. Natl Inst. Stand. Technol. 114, 341-358 (2009).

53. Boekholt, $M$. et al. Determination of the sound velocities and the complete set of elastic constants for $\mathrm{Bi}_{2} \mathrm{Sr}_{2} \mathrm{CaCu}_{2} \mathrm{O}_{8+\delta}$ single crystals using Brillouin light scattering. Physica C 179, 101-106 (1991).

54. Kresse, G. \& Furthmüller, J. Efficient iterative schemes for ab initio totalenergy calculations using a plane-wave basis set. Phys. Rev. B 54, 11169 (1996).

55. Kresse, G. \& Joubert, D. From ultrasoft pseudopotentials to the projector augmented-wave method. Phys. Rev. B 59, 1758 (1999).

56. Perdew, J. P., Burke, K. \& Ernzerhof, M. Generalized gradient approximation made simple. Phys. Rev. Lett. 78, 3865 (1996).

57. Togo, A. \& Tanaka, I. First principles phonon calculations in materials science. Scr. Mater. 108, 1-5 (2015).

58. Silvi, B. \& Savin, A. Classification of chemical bonds based on topological analysis of electron localization functions. Nature 371, 683-686 (1994).

\section{Acknowledgements}

This work was supported by the National Natural Science Foundation of China (Grant nos. 11934007 and 11804346), the Key Research Program of Frontier Sciences, Chinese Academy of Sciences (Grant no. ZDBS-LY-JSC002), and the Liaoning Revitalization Talents Program (Grant no. XLYC1807122). J.S.Z. was supported by an NSF grant (MRSEC DMR-1720595). We acknowledge beam time awarded by ANSTO (Proposal no. P7373), ORNL (Proposal no. IPTS-21435.1), and SPring-8 (Proposal no. 2019A1249). A portion of this research used resources at Spallation Neutron Source, a DOE Office of Science User Facility operated by the Oak Ridge National Laboratory. We thank Dr. Richard Mole for the help on the onsite data reduction and crystal alignment at Pelican as well as Dr. Guochu Deng for pre-aligning the crystal at the Joey Neutron Laue Camera.

\section{Author contributions}

B.L. and Zhidong Zhang conceived the idea. J.Q., Zongteng Zhang, and W.R. synthesized the single crystals. X.C., Jiaming He, and J.Z. measured the thermal conductivity of single crystals while L.F. and Jiaqing He measured the thermal conductivity of the polycrystalline pellet. Q.Z. collected the neutron powder diffraction data. X.J. collected the single-crystal X-ray diffraction data and did the structural refinements. Y.C., K.O., S.H., and O.S. performed the synchrotron X-ray diffraction and PDF analysis. Zhe Zhang, Zhao Zhang, B.L., D.Y., and S.D. conducted the INS measurements. G.C. performed the Brillouin light scattering experiments and determined the velocity. B.D. and T.Y. performed the DFT calculations. J.Q. analyzed the data and wrote the paper with B.L. and D.Y.

\section{Competing interests}

The authors declare no competing interests.

\section{Additional information}

Supplementary information is available for this paper at https://doi.org/10.1038/s41467020-19044-w.

Correspondence and requests for materials should be addressed to D.Y. or B.L.

Peer review information Nature Communications thanks Qingyu Yan and the other, anonymous, reviewer(s) for their contribution to the peer review of this work.

Reprints and permission information is available at http://www.nature.com/reprints

Publisher's note Springer Nature remains neutral with regard to jurisdictional claims in published maps and institutional affiliations.

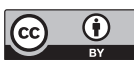

Open Access This article is licensed under a Creative Commons Attribution 4.0 International License, which permits use, sharing, adaptation, distribution and reproduction in any medium or format, as long as you give appropriate credit to the original author(s) and the source, provide a link to the Creative Commons license, and indicate if changes were made. The images or other third party material in this article are included in the article's Creative Commons license, unless indicated otherwise in a credit line to the material. If material is not included in the article's Creative Commons license and your intended use is not permitted by statutory regulation or exceeds the permitted use, you will need to obtain permission directly from the copyright holder. To view a copy of this license, visit http://creativecommons.org/ licenses/by/4.0/.

(c) The Author(s) 2020 